\title{
A PAPKOVICH-NEUBER-BASED NUMERICAL APPROACH TO CRACKS WITH CONTACT IN 3D
}

\author{
M. HINTERMÜLLER ${ }^{1,2}$, V.A. KOVTUNENKO ${ }^{1,3}$, AND K. KUNISCH $^{1}$
}

\begin{abstract}
The mathematical model of a crack with non-penetration conditions is considered in the framework of three-dimensional elasticity. The spatial crack problem is investigated with respect to its numerical realization in the context of constrained optimization. Specifically, for homogeneous isotropic solids with planar cracks, a Papkovich-Neuber-based representation is adopted. It allows to employ a primal-dual active-set strategy with an unconditional global and monotone convergence property. The iterates turn out to be primally feasible. Illustrative numerical examples are presented.
\end{abstract}

\section{Motivation And Introduction}

In the framework of three-dimensional elasticity theory we consider a mathematical model of cracks constrained by non-penetration conditions, which allow the contact of opposite crack faces, but not their penetration. Properties of well-posedness for such problems were studied in terms of variational methods by (Khludnev and Sokolowski 1999, Khludnev and Kovtunenko 2000). In the present paper we investigate a numerical approach to the constrained crack problems. Our considerations focus on three-dimensional homogeneous isotropic solids with planar cracks. For three-dimensional aspects of fracture mechanics we refer to the classic concepts as presented by (Cherepanov 1979, Geubelle and Rice 1995, Morozov and Petrov 2000), and to their numerical treatment by (Aliabadi and Rooke 1991, Stavroulakis 2001).

Before starting with a detailed description, let us present a few illustrative examples motivating on one hand the consideration of nonpenetration conditions for a crack and, on the other hand, the need

1991 Mathematics Subject Classification. 49J40, 49M29, 73M05, 73C99.

Key words and phrases. Crack with non-penetration, constrained optimization problem, primal-dual active-set algorithm, Papkovich-Neuber representation, numerical calculation.

${ }^{1}$ Department of Mathematics, University of Graz, Graz, Austria.

${ }^{2}$ Chair in Applied Mathematics, University of Sussex, Brighton, U.K..

3 Lavrent'ev Institute of Hydrodynamics, Novosibirsk, Russia. 
of a fully three-dimensional formulation. With respect to the latter aspect, in the present context we point out that we cannot expect a physically meaningful decoupling of the model into independent planar states, in general. Further, the assumption of stress-free crack faces is not applicable due to the specific loading and the spatial nature of the problems under consideration. Indeed, contact of crack faces is possible and gives rise to a non-zero normal stress (the contact force) at the crack faces. In the following we illustrate these facts by means of numerical examples.

We consider the following rectangular geometry of a solid with a crack in the unit cube $\left\{0<x_{1}<1,0<x_{2}<1,\left|x_{3}\right|<0.5\right\}$. The solid is clamped at $x_{1}=1$ and it is loaded by a traction force applied uniformly at the marked part of the boundary; see Figure 1 (a). Assuming the condition of mutual non-penetration for vertical displacements (along the $x_{3}$-axis) between the opposite surfaces of the crack in the $\left\{x_{3}=0\right\}$-plane, we compute a numerical solution of the elasticity problem. We find a contact zone of the crack surfaces which is indicated in black in Figure 1 (b). Following the standard terminology adopted in constrained optimization, this zone is referred to as the active set. The components of displacements and stresses at both crack surfaces are depicted respectively in Figure 2. This example clearly illustrates the known influence of the boundary at $x_{2}=0$ and $x_{2}=1$, that restricts the applicability of planar models.

In the following examples 2 and 3 we keep the above geometry of the solid with crack and change the loading only. The configuration of Example 2 is shown in Figure 3 (a). The solution of the corresponding elasticity problem is depicted in a componentwise fashion in Figure 4. We can see in Figure 3 (b) that the active (contact) zone is split into two separate sets, which exhibit no symmetry. Hence, planar models cannot be applied here.

Note that, due to the symmetry of the solid and the loading with respect to the $\left\{x_{3}=0\right\}$-plane, these two examples have zero jumps of tangential displacements across the crack (see the 1- and 2-components of $\boldsymbol{u}$ in Figure 2 and Figure 4) and a non-negative jump (opening) of the vertical displacement (i.e., the 3-component in Figure 2 and Figure 4), thus implying a mode- 1 state of the crack. 
(a) solid with crack

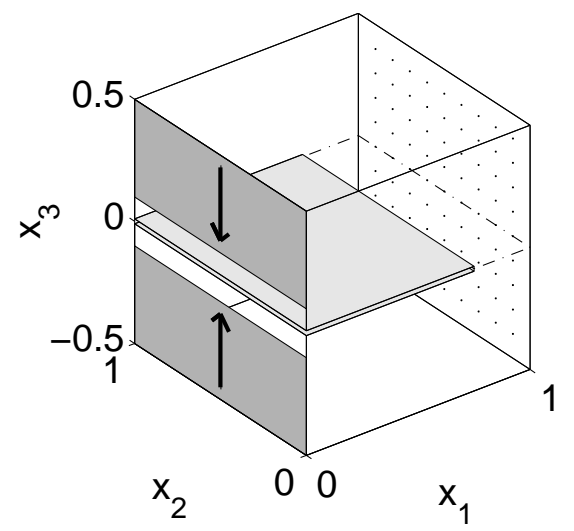

(b) contact of crack surfaces

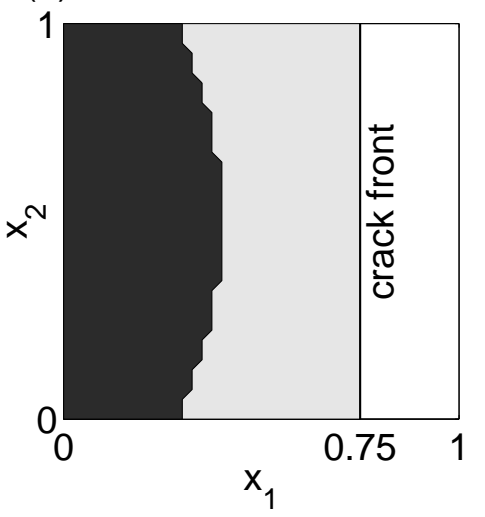

Figure 1. Example 1: geometry and loading in the crack problem.
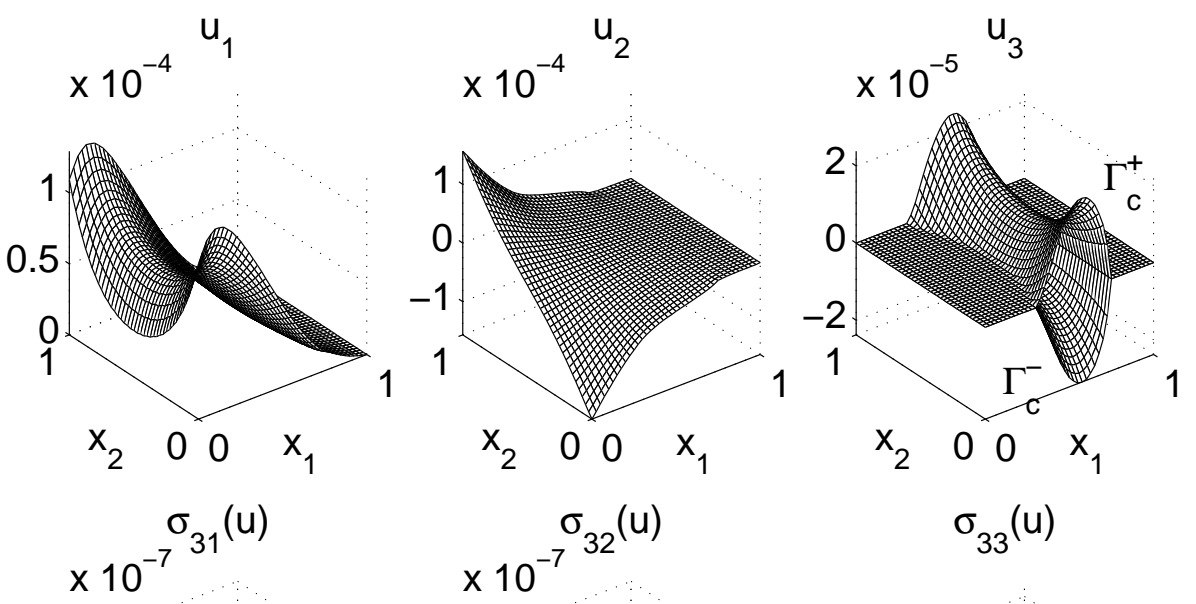

$\times 10^{-\sigma_{32}(u)}$

$$
\sigma_{33}(u)
$$
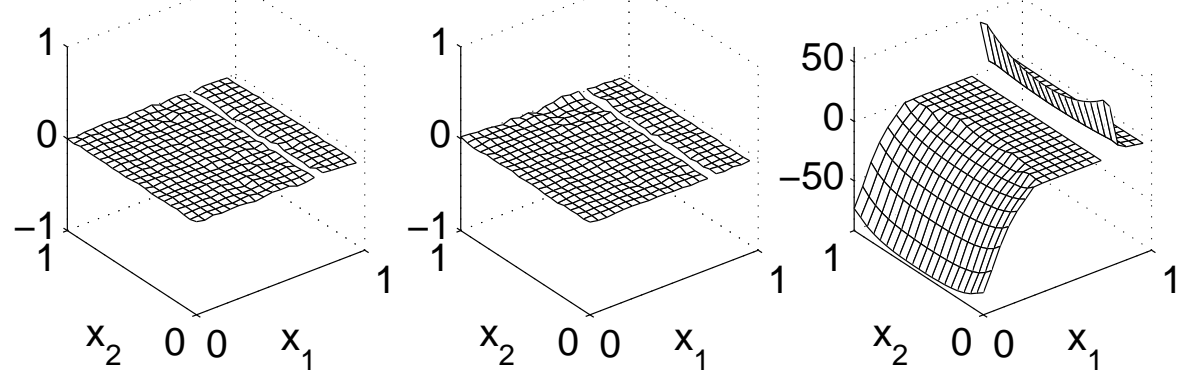

Figure 2. Example 1: displacement and stress at the crack.

The last example illustrated in Figure 5 (a) deals with the case of the absence of a symmetry with respect to the $\left\{x_{3}=0\right\}$-plane. As 
(a) solid with crack

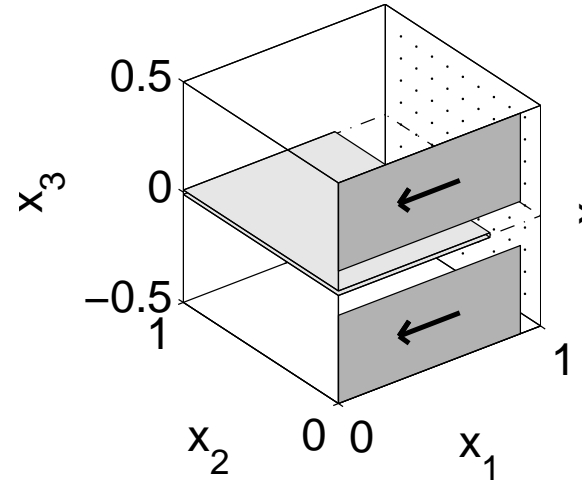

(b) contact of crack surfaces

Figure 3. Example 2: geometry and loading in the crack problem.
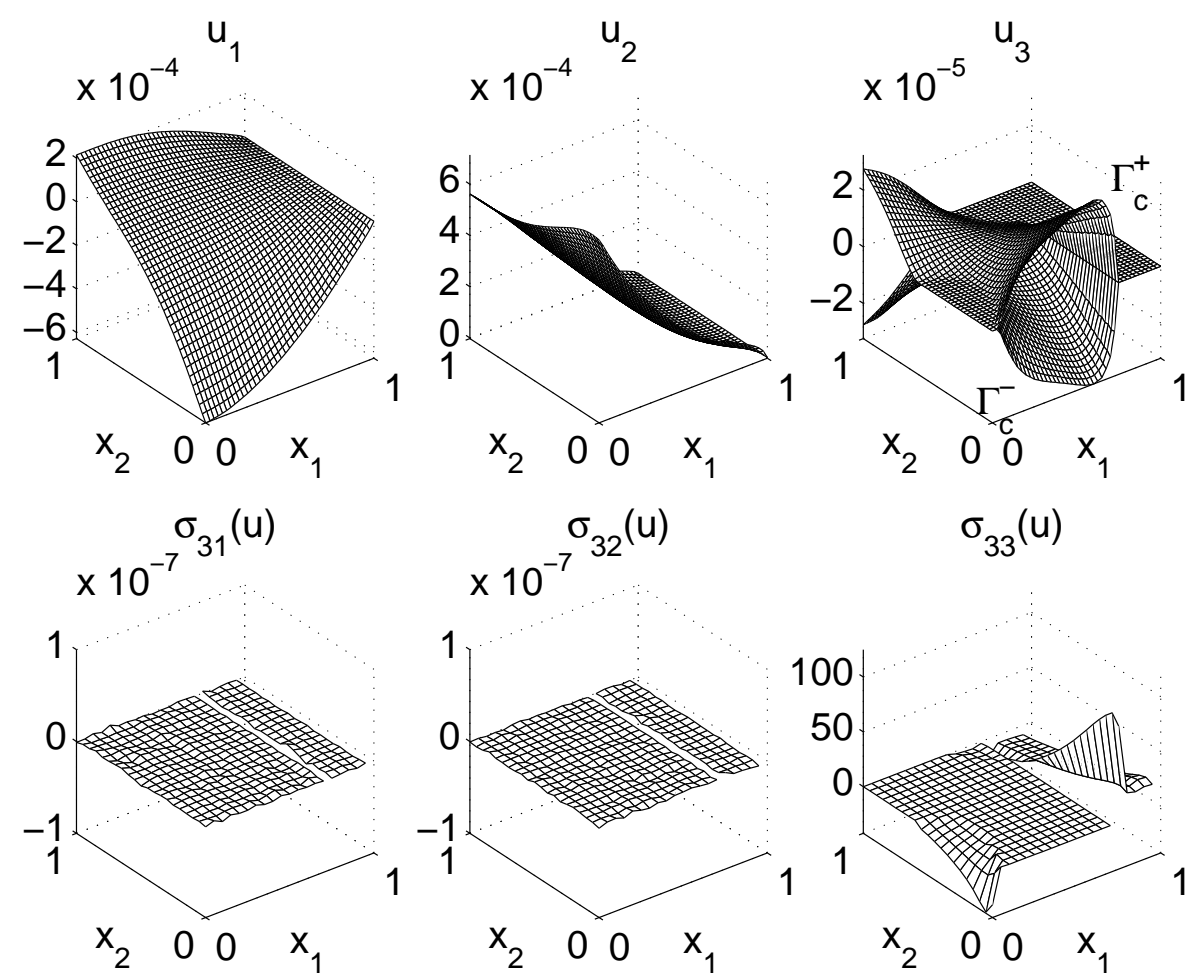

FiguRE 4. Example 2: displacement and stress at the crack.

a consequence, in Figure 6 we can clearly see the appearance of a 
(a) solid with crack

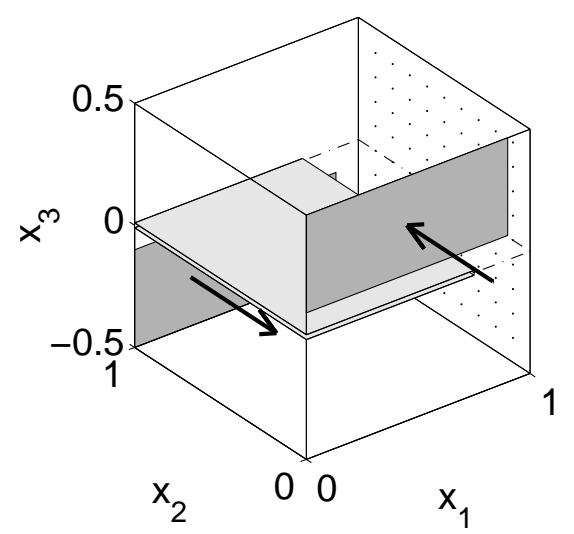

(b) contact of crack surfaces

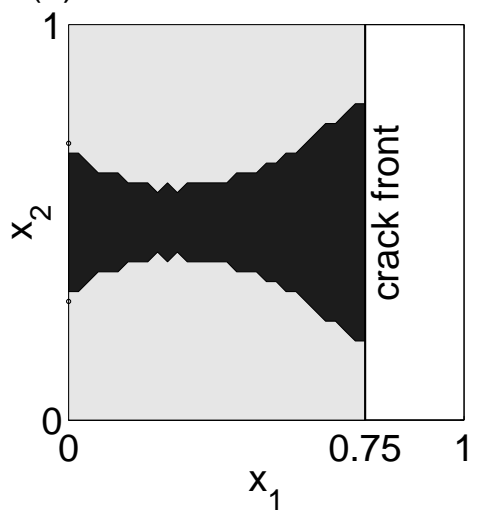

Figure 5. Example 3: geometry and loading in the crack problem.
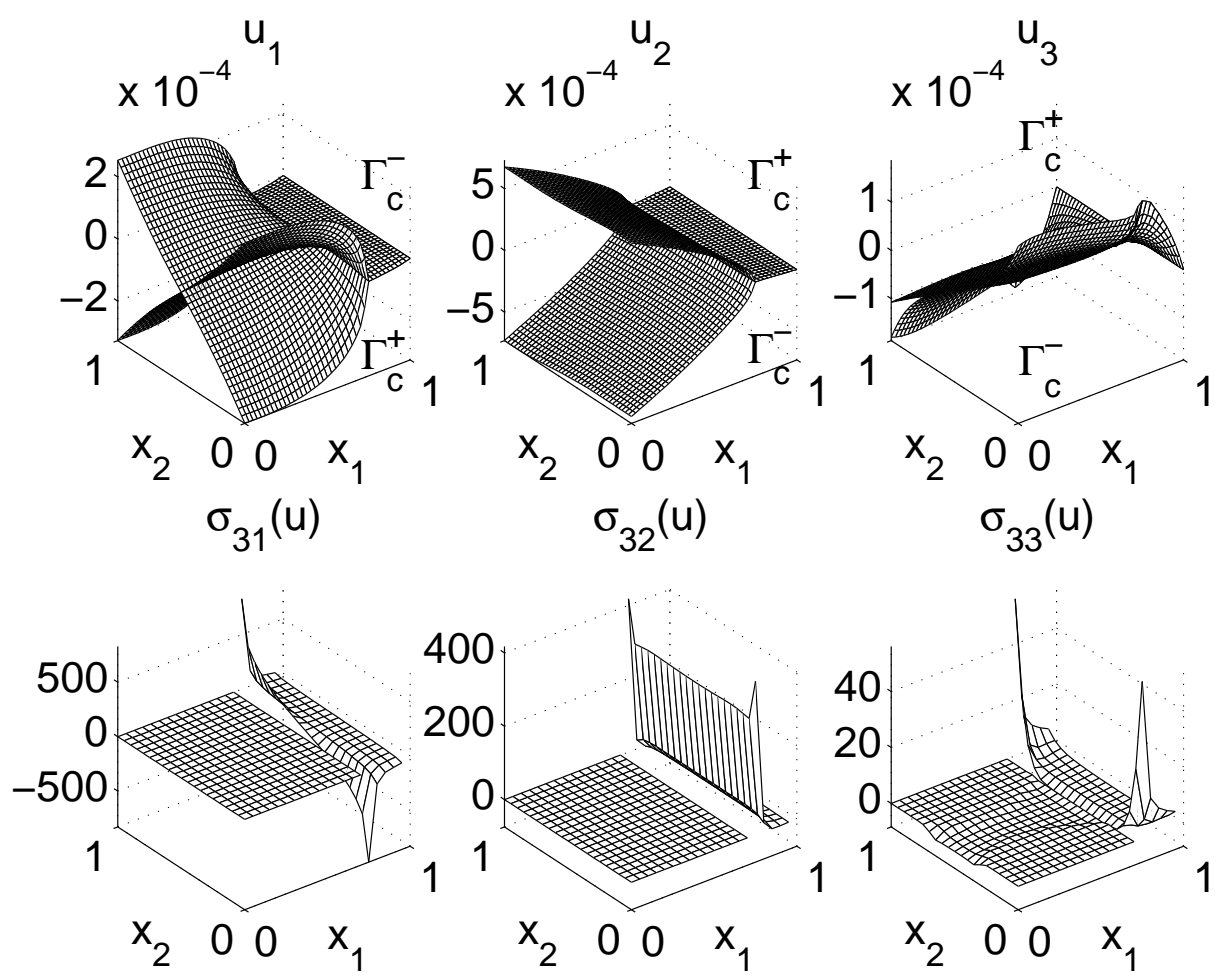

Figure 6. Example 3: displacement and stress at the crack.

mixed state of mode- 1 , mode- 2 , and mode- 3 of the crack, i.e. nonzero jumps for all displacement components across the crack. The 
active (contact) set forms an axis-symmetric region with respect to the $\left\{x_{2}=0.5, x_{3}=0\right\}$-axis in Figure $5(\mathrm{~b})$.

In the classical fracture mechanics context, one of the most intriguing aspects is to determine the stress intensity factors in three spatial dimensions. For its various theoretical and numerical concepts we refer to (Nikishkov and Atluri 1987, Kachanov and Karapetian 1997, Gosz, Dolbow and Moran 1998). The stress intensity factors $\left(K_{1}, K_{2}, K_{3}\right)$ will help us to compare the obtained numerical results with the ones derived for the linearized model assuming stress-free crack faces. For this reason we adopt an approximate formula, see (Cisilino and Aliabadi 1999), which is useful in engineering practice:

$$
\left(K_{1}, K_{2}, K_{3}\right)=\frac{E}{4\left(1-\nu^{2}\right)} \sqrt{\frac{\pi}{2 h}}\left(\llbracket u_{3}^{h} \rrbracket, \llbracket u_{1}^{h} \rrbracket,(1-\nu) \llbracket u_{2}^{h} \rrbracket\right) .
$$

The jumps $\left(\llbracket u_{1}^{h} \rrbracket, \llbracket u_{2}^{h} \rrbracket, \llbracket u_{3}^{h} \rrbracket\right)$ of the displacement components are evaluated at a distance $h$ behind the crack front. The material parameters $E$ and $\nu$ stand for the Young's modulus and the Poisson ratio.

When the loading is chosen such that it prevents self-penetration of crack faces, validation of our numerical tools was given in (Kovtunenko 2006b). The results were compared with an exact three-dimensional solution which obeys the known square-root singularities. The example configuration of the present paper forces contact between the crack faces. As a consequence, the difference between models occurs when we take into account non-penetration conditions or ignore them in the model.

First, the result of numerical calculation of the stress intensity factors by formula (1.1) is depicted in Figure 7 for the geometry and the loading given in the examples 1 and 2 . Since $\llbracket u_{1}^{h} \rrbracket=0$ and $\llbracket u_{2}^{h} \rrbracket=0$ along the crack front, then $K_{2}=K_{3}=0$ in these examples. Hence, only the non-zero $K_{1}$ is shown. In Figure 7 (a) $K_{1}$ is calculated for the constrained crack model which prevents self-penetration. Respectively, in Figure 7 (b) $K_{1}$ is given for the linearized model admitting penetration of the crack faces. The negative stress intensity factor $K_{1}$ in Figure 7 (b) implies self-penetration of crack faces which is inconsistent physically. It can be seen that due to the prescribed compression loading the observed error of the linearized setting of the crack problem is tremendous in the example 1 . The quantity $K_{1}$ along the crack front in example 2 is depicted in Figure 7 (c) for both models simultaneously. The dashed line corresponds to the model with penetration, whereas the solid line reflects the model without penetration. The figure shows the significant difference between the curves which implies that the assumption of stress-free crack faces is too coarse in this case. 
Secondly, in the mixed-mode state of example 3, all three stress intensity factors $\left(K_{1}, K_{2}, K_{3}\right)$ are non-zero, and they are depicted in Figure 8. While two of them, $K_{2}$ and $K_{3}$, visually coincide for the linearized and the constrained setting of the crack problem, the difference for $K_{1}$ attains a relative error of $47 \%$. Even if we cut off the negative values of $K_{1}$ at zero, an error of $21 \%$ remains for the linearized setting.

It is interesting to note, that the difference of the stress intensity factors occurs only for $K_{1}$ associated to the constrained component of the solution of the crack problem.

(a) Example 1

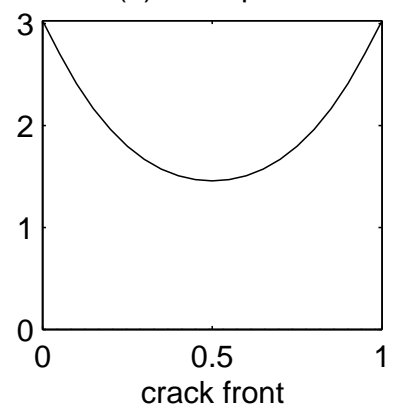

(b) Example 1

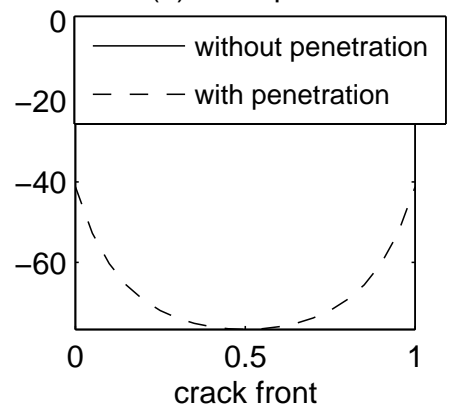

(c) Example 2

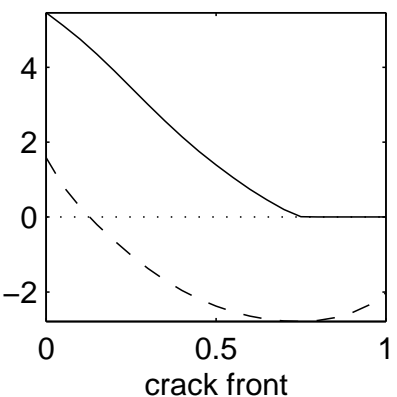

Figure 7. The non-zero stress intensity factor $K_{1}$ $(\mathrm{mPa} \sqrt{\mathrm{m}})$ at the crack front in Examples 1 and 2 .
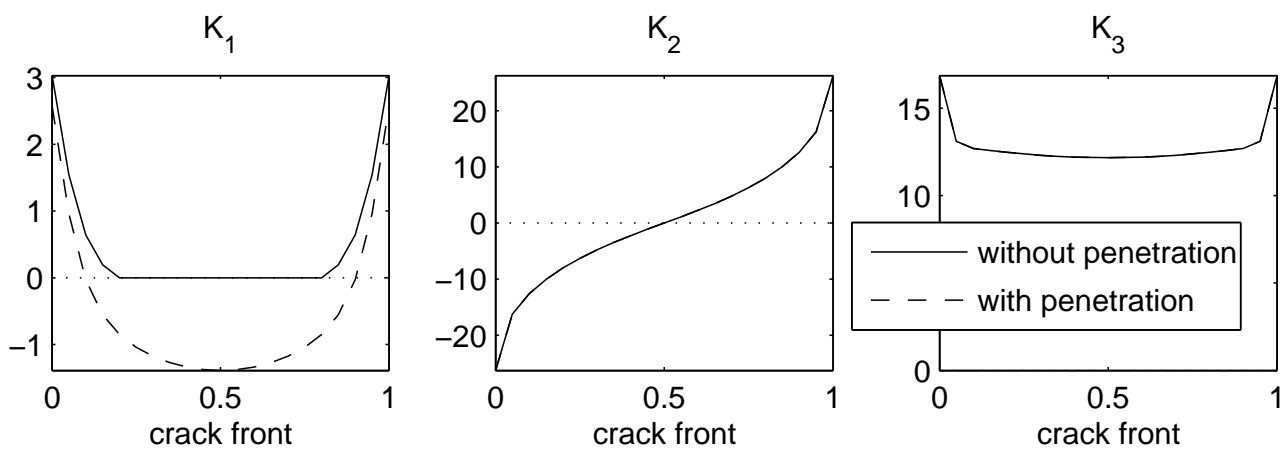

Figure 8. The mixed-mode stress intensity factors $K_{1}$, $K_{2}$, and $K_{3}(\mathrm{mPa} \sqrt{\mathrm{m}})$ at the crack front in Example 3.

For computing a solution of crack problems with non-penetration conditions we apply the so-called primal-dual active-set strategy, which is based on a semi-smooth differentiability property of the operator of the problem; see (Ito and Kunisch 2003, Hintermüller, Ito and Kunisch 2003). It is a very efficient tool to solve constrained optimization 
problems. The reason lies in the fact that a primal state variable (displacement) and a dual state variable (normal stress) are used simultaneously to determine an active (contact) set properly, even in cases when the two variables are close to zero. Compared to, e.g., purely primal approaches which are based on $\boldsymbol{u}$ only, our primal-dual technique is numerically more stable. In fact, it typically avoids possible chattering phenomena of the algorithm due to primal degeneracy, i.e., very flat transitions of the jump of the displacement across the crack into the active set. In the present paper we investigate global convergence properties of this strategy based on a Papkovich-Neuber representation in three-dimensional elasticity.

From a general point of view, the principal difficulty of a convergence analysis of the primal-dual algorithm concerns here the absence of a maximum principle for vector-valued coupled systems. Therefore, we utilize asymptotic arguments based on a Papkovich-Neuber representation of the three-dimensional Lamé-equations via harmonic potentials, which obey the required maximum principle. Let us briefly recall the main formulas; see (Goldstein and Entov 1994) for details. For the vector-valued Lamé-equation in the space $\left\{\boldsymbol{x} \in \mathbb{R}^{3}\right\}$, e.g.,

$$
-\mu \Delta \boldsymbol{v}-(\mu+\lambda) \nabla(\operatorname{div} \boldsymbol{v})=\boldsymbol{f}
$$

the solution can be represented as

$$
\boldsymbol{v}=\frac{1}{\mu} \boldsymbol{\psi}-\frac{\kappa}{2 \mu} \nabla\left(\boldsymbol{x}^{\top} \boldsymbol{\psi}-\phi\right), \quad \kappa=\frac{\mu+\lambda}{2 \mu+\lambda},
$$

by the functions $\boldsymbol{\psi}=\left(\psi_{1}, \psi_{2}, \psi_{3}\right)$ and $\phi$ satisfying

$$
-\Delta \boldsymbol{\psi}=\boldsymbol{f}, \quad-\Delta \phi=\boldsymbol{x}^{\top} \boldsymbol{f} .
$$

Here $\mu>0$ and $\lambda>0$ denote the Lamé parameters and $\boldsymbol{f}$ is a volume force. For $\boldsymbol{f}=\mathbf{0}$ and with the particular choice

$$
\psi_{\alpha}=\mu(\kappa-1) U_{\alpha}(\alpha=1,2), \quad \psi_{3}=\mu(\kappa+1) U_{3}, \quad \phi=\mu(\kappa-1) \boldsymbol{x}^{\top} \boldsymbol{U}
$$

in (1.4), where $\boldsymbol{U}$ satisfies

$$
\Delta \boldsymbol{U}=\mathbf{0}, \quad \operatorname{div} \boldsymbol{U}=0 \quad \text { (for example } \boldsymbol{U}=\nabla \Phi, \quad \Delta \Phi=0),
$$

the representation (1.3) reads

$$
v_{\alpha}=(\kappa-1) U_{\alpha}-\kappa x_{3} U_{3, \alpha}(\alpha=1,2), \quad v_{3}=U_{3}-\kappa x_{3} U_{3,3} .
$$

The relations (1.6) imply a decoupling of the third components $v_{3}$ of the variables from $\left(v_{1}, v_{2}\right)$ at the $\left\{x_{3}=0\right\}$-plane:

$$
v_{3}=U_{3}, \quad \sigma_{33}(\boldsymbol{v}):=2 \mu v_{3,3}+\lambda \operatorname{div} \boldsymbol{v}=2 \mu \kappa U_{3,3} \quad \text { at } x_{3}=0 .
$$


As a consequence of (1.5) and (1.7), positivity/ negativity properties of $v_{3}$ and $\sigma_{33}(\boldsymbol{v})$ are determined from the following Hopf (strong) maximum principle for the harmonic function $U_{3}$ :

The derivative $U_{3,3}$ (hence $\sigma_{33}(\boldsymbol{v})$ ) is strictly negative/ positive at the maximum/ minimum of $U_{3}$ (hence $v_{3}$ ) at the boundary.

Employing these well-known constructions we are able to define a primal-dual active-set strategy with an unconditional global and monotone convergence property in Section 2. Section 3 is devoted to computational features of our numerical algorithm for the examples presented at the beginning of this section.

We remark that a purely primal numerical method for constrained crack problems is presented in (Zozulya and Menshykov 2003). For advanced discretization techniques we refer to the meshless finite-element methods modelling three-dimensional cracks as developed by (Sukumar, Moës, Moran and Belytschko 2000), and to the adaptive finite elements in contact problems by $\mathrm{(Hu}$, Panagiotopoulos, Panagouli, Scherf and Wriggers 2000).

\section{Continuous setting of the Problem}

\subsection{The mixed boundary value formulation of a constrained}

crack problem. Let $\Gamma_{C}$ be a planar crack posed on the plane $\left\{x_{3}=0\right\}$ and located inside a domain $\Omega \subset \mathbb{R}^{3}$ with the boundary $\partial \Omega=\Gamma$. The crack $\Gamma_{C}$ is assumed to be an open set in the plane $\left\{x_{3}=0\right\}$. For the vector $\boldsymbol{u}=\left(u_{1}, u_{2}, u_{3}\right)^{\top}(\boldsymbol{x})$ of displacements of a point $\boldsymbol{x}=$ $\left(x_{1}, x_{2}, x_{3}\right)^{\top} \in \mathbb{R}^{3}$ we introduce the standard tensors of three-dimensional elasticity for the stress and strain as

$$
\begin{aligned}
& \sigma_{i j}(\boldsymbol{u})=2 \mu \varepsilon_{i j}(\boldsymbol{u})+\lambda \delta_{i j} \operatorname{div} \boldsymbol{u}, \\
& \varepsilon_{i j}(\boldsymbol{u})=0.5\left(u_{i, j}+u_{j, i}\right) \quad(i, j=1,2,3),
\end{aligned}
$$

with the Lamé parameters $\mu$ and $\lambda$. For a given load $\boldsymbol{f}=\left(f_{1}, f_{2}, f_{3}\right)^{\top}(\boldsymbol{x})$ we consider the following problem of an equilibrium in $\Omega_{C}=\Omega \backslash \Gamma_{C}$ of a solid with the crack subject to non-penetration conditions written in the strong form:

$$
\begin{gathered}
-\mu \Delta \boldsymbol{u}-(\mu+\lambda) \nabla(\operatorname{div} \boldsymbol{u})=\boldsymbol{f} \quad \text { in } \Omega_{C}, \\
\boldsymbol{u}=\mathbf{0} \quad \text { on } \Gamma, \\
\sigma_{13}(\boldsymbol{u})=\sigma_{23}(\boldsymbol{u})=0 \quad \text { on } \Gamma_{C}^{ \pm}, \\
\llbracket u_{3} \rrbracket \geq 0, \quad \sigma_{33}(\boldsymbol{u}) \leq 0, \quad \sigma_{33}(\boldsymbol{u}) \llbracket u_{3} \rrbracket=0 \quad \text { on } \Gamma_{C},
\end{gathered}
$$

with the jump $\llbracket u_{3} \rrbracket=\left.u_{3}\right|_{\Gamma_{C}^{+}}-\left.u_{3}\right|_{\Gamma_{C}^{-}}$across the crack surfaces $\Gamma_{C}^{ \pm}$. 
The weak formulation of (2.2) as a variational inequality, i.e.,

$$
\int_{\Omega_{C}} \sigma_{i j}(\boldsymbol{u}) \varepsilon_{i j}(\boldsymbol{v}-\boldsymbol{u}) d x \geq \int_{\Omega_{C}} f_{i}(v-u)_{i} d s \quad \text { for all } \boldsymbol{v} \in K
$$

where

$$
K=\left\{\boldsymbol{v} \in H^{1}\left(\Omega_{C}\right)^{3}: \quad \boldsymbol{v}=\mathbf{0} \quad \text { on } \Gamma, \quad \llbracket v_{3} \rrbracket \geq 0 \quad \text { on } \Gamma_{C}\right\},
$$

provides us with the generalized solution $\boldsymbol{u} \in K$ to (2.2). The solution satisfies the complementarity conditions $(2.2 \mathrm{~d})$ in the weak form:

$$
\llbracket u_{3} \rrbracket \geq 0 \quad \text { on } \Gamma_{C}, \quad\left\langle\sigma_{33}(\boldsymbol{u}), \llbracket v_{3}-u_{3} \rrbracket\right\rangle_{\Gamma_{C}} \leq 0 \quad \text { for all } \boldsymbol{v} \in K .
$$

The notation $\langle\cdot, \cdot\rangle_{\Gamma_{C}}$ stands for the duality pairing between elements of the mutual dual spaces $H^{1 / 2}$ and $H^{-1 / 2}$ at $\Gamma_{C}$; see (Khludnev and Kovtunenko 2000) for a detailed description of the dual spaces of traces at the crack. In the above reference additional local $\mathrm{H}^{2}$-regularity of the solution is established except at the crack front. As a consequence, $\sigma_{33}(\boldsymbol{u}) \in H_{l o c}^{1 / 2}\left(\Gamma_{C}\right)$, i.e., for any $\overline{\boldsymbol{x}} \in \Gamma_{C}, \overline{\boldsymbol{x}}=\left(x_{1}, x_{2}\right)^{\top}$, there exists a neighborhood $O(\overline{\boldsymbol{x}}) \subset \Gamma_{C}$ such that $\sigma_{33}(\boldsymbol{u}) \in H^{1 / 2}(O(\overline{\boldsymbol{x}}))$. In particular, $\sigma_{33}(\boldsymbol{u})$ is almost everywhere well defined in $\Gamma_{C}$.

The complementarity condition $(2.2 \mathrm{~d})$ can therefore be expressed equivalently as mixed boundary conditions, with equalities holding in the almost everywhere sence, as follows:

$$
\llbracket u_{3} \rrbracket=0 \quad \text { on } A, \quad \sigma_{33}(\boldsymbol{u})=0 \quad \text { on } I:=\Gamma_{C} \backslash A,
$$

where the strongly active set $A \subseteq \Gamma_{C}$ is defined by

$$
A=\left\{\overline{\boldsymbol{x}} \in \Gamma_{C}: \quad\left(c \sigma_{33}(\boldsymbol{u})+\llbracket u_{3} \rrbracket\right)(\overline{\boldsymbol{x}}, 0)<0\right\},
$$

where $c>0$ is an arbitrary constant. The complementary inactive set $I$ is given by

$$
I=\left\{\overline{\boldsymbol{x}} \in \Gamma_{C}: \quad\left(c \sigma_{33}(\boldsymbol{u})+\llbracket u_{3} \rrbracket\right)(\overline{\boldsymbol{x}}, 0) \geq 0\right\},
$$

which also contains the weakly active set

$$
\left\{\overline{\boldsymbol{x}} \in \Gamma_{C}: \quad\left(c \sigma_{33}(\boldsymbol{u})+\llbracket u_{3} \rrbracket\right)(\overline{\boldsymbol{x}}, 0)=0\right\} .
$$

For properties of the equivalence between mixed formulations and variational inequalities see (Kovtunenko 2006a).

In the following section a primal-dual active set strategy suggested by the mixed formulation (2.3)-(2.4) will be analyzed. At each iteration level $n=-1,0, \ldots$ a problem of the type $(2.2 \mathrm{a})-(2.2 \mathrm{c})$ will be solved with the additional constraints that $\llbracket u_{3}^{(n+1)} \rrbracket=0$ on the currently strongly active set $A^{(n)}$ and $\sigma_{33}\left(\boldsymbol{u}^{(n+1)}\right)=0$ on the complement of $A^{(n)}$. Such a procedure can be very efficient numerically. Its mathematical 
analysis is complicated by the fact that the solution of the intermediate problems $\boldsymbol{u}^{(n+1)}$ can be less regular than the solution $\boldsymbol{u}$ of the limit problem. In fact $\sigma_{33}\left(\boldsymbol{u}^{(n+1)}\right)$ belongs to $H_{l o c}^{-1 / 2}\left(\Gamma_{C}\right)$ with singularities along the boundary of $A^{(n)}$. In previous work regularization by penalization or by discretization was considered. After regularization, the primal-dual active set strategy, which is equivalent to a semi-smooth Netwon method, is used. Its local convergence analysis follows from (Hintermüller et al. 2003, Ito and Kunisch 2003). However, a monotone global convergence property is available only in specific cases; see (Hintermüller, Kovtunenko and Kunisch 2004) for examples.

In the present work we investigate the primal dual active set strategy without regularization. We shall require, instead, an assumption on the regularity of the active sets $A^{(n)}$ of the intermediate problems, which will allow a pointwise almost every interpretation of $\llbracket u_{3}^{(n+1)} \rrbracket$ and $\sigma_{33}\left(\boldsymbol{u}^{(n+1)}\right)$. We shall further rely on a formulation of the constrained crack problem in $\mathbb{R}^{3}$-space by asymptotic arguments. Using the Papkovich-Neuber representation for a solution of the mixed problem of linear elasticity in a half-space, we prove global and monotone convergence properties of the iterates of the primal-dual active set strategy in $\mathbb{R}^{3}$. This property is confirmed by our numerical findings in Section 3 .

2.2. The primal-dual active set strategy in $\mathbb{R}^{3}$-space. In this section we neglect the influence of the external boundary $\Gamma$ and consider the problem on all of $\mathbb{R}^{3}$. This relates to the situation where we concentrate on the behavior of the system (2.2) in a neighborhood of the crack. We investigate the following problem on $\mathbb{R}^{3} \backslash \Gamma_{C}$ :

$$
\begin{aligned}
& -\mu \Delta \boldsymbol{u}-(\mu+\lambda) \nabla(\operatorname{div} \boldsymbol{u})=\boldsymbol{f} \quad \text { in } \mathbb{R}^{3} \backslash \Gamma_{C}, \\
& \boldsymbol{u}(\boldsymbol{x})=\boldsymbol{o}(1) \quad \text { as }|\boldsymbol{x}| \rightarrow \infty, \\
& \sigma_{13}(\boldsymbol{u})=\sigma_{23}(\boldsymbol{u})=0 \quad \text { on } \Gamma_{C}^{ \pm}, \\
& \llbracket u_{3} \rrbracket \geq 0, \quad \sigma_{33}(\boldsymbol{u}) \leq 0, \quad \sigma_{33}(\boldsymbol{u}) \llbracket u_{3} \rrbracket=0 \quad \text { on } \Gamma_{C} .
\end{aligned}
$$

Since $\sigma_{33}(\boldsymbol{u})$ has a pointwise almost everywhere meaning in the open set $\Gamma_{C}$, the complemantarity condition in (2.5), i.e., the last three relations, can be stated as mixed boundary conditions with respect to the active set $A$ and its inactive complement $I$ in $\Gamma_{C}$ :

$$
\begin{aligned}
& \llbracket u_{3} \rrbracket=0 \quad \text { on } A=\left\{\overline{\boldsymbol{x}} \in \Gamma_{C}:\left(c \sigma_{33}(\boldsymbol{u})+\llbracket u_{3} \rrbracket\right)(\overline{\boldsymbol{x}}, 0)<0\right\}, \\
& \sigma_{33}(\boldsymbol{u})=0 \quad \text { on } I:=\Gamma_{C} \backslash A .
\end{aligned}
$$

We define a primal-dual active set algorithm corresponding to (2.5).

\section{Algorithm 1.}


(0) Choose $A^{(-1)} \subset \mathbb{R}^{2}$; set $n=-1$.

(1) Solve for $\boldsymbol{u}^{(n+1)}$ :

$$
\begin{aligned}
& -\mu \Delta \boldsymbol{u}^{(n+1)}-(\mu+\lambda) \nabla\left(\operatorname{div} \boldsymbol{u}^{(n+1)}\right)=\boldsymbol{f} \quad \text { in } \mathbb{R}^{3} \backslash \Gamma_{C}, \\
& \boldsymbol{u}^{(n+1)}(\boldsymbol{x})=\boldsymbol{o}(1) \quad \text { as }|\boldsymbol{x}| \rightarrow \infty, \\
& \sigma_{13}\left(\boldsymbol{u}^{(n+1)}\right)=\sigma_{23}\left(\boldsymbol{u}^{(n+1)}\right)=0 \quad \text { on } \Gamma_{C}^{ \pm}, \\
& \llbracket u_{3}^{(n+1)} \rrbracket(\overline{\boldsymbol{x}}, 0)=0 \quad \text { for } \overline{\boldsymbol{x}} \in A^{(n)}, \\
& \sigma_{33}\left(\boldsymbol{u}^{(n+1)}\right)(\overline{\boldsymbol{x}}, 0)=0 \quad \text { for } \overline{\boldsymbol{x}} \in I^{(n)}:=\Gamma_{C} \backslash A^{(n)} .
\end{aligned}
$$

(2) Compute the active set:

$$
A^{(n+1)}=\left\{\overline{\boldsymbol{x}} \in \Gamma_{C}:\left(c \sigma_{33}\left(\boldsymbol{u}^{(n+1)}\right)+\llbracket u_{3}^{(n+1)} \rrbracket\right)(\overline{\boldsymbol{x}}, 0)<0\right\} .
$$

(3) If $A^{(n+1)}=A^{(n)}$ then STOP; else set $n=n+1$ and go to Step 1.

In the following we rely on the assumption which guarantees that the iterates $\sigma_{33}\left(\boldsymbol{u}^{(n+1)}\right)$ are defined almost everywhere in $\Gamma_{C}$. This, in turn, will be implied by an assumption that at each iteration level the interface between strongly active $A^{(n)}$ and inactive $I^{(n)}$ sets is sufficiently regular so that $\sigma_{33}\left(\boldsymbol{u}^{(n+1)}\right) \in H_{l o c}^{1 / 2}\left(A^{(n)}\right)$.

Lemma 2.1. For all $n=-1,0, \ldots$, we assume that $A^{(n)}=\bigcup_{i=0}^{\infty} A_{i}^{(n)}$, where $A_{i}^{(n)}=\stackrel{\circ}{A}_{i}^{(n)} \subset \mathbb{R}^{2}$ are pairwise disjoint sets of meas $\left(A_{i}^{(n)}\right) \neq 0$ for $i=1, \ldots, \infty$, and meas $\left(A_{0}^{(n)}\right)=0$ in $\mathbb{R}^{2}$. If the respective boundaries of $A_{i}^{(n)} \cap \Gamma_{C}$ are sufficiently smooth for $i=1, \ldots, \infty$, then the normal stress $\sigma_{33}\left(\boldsymbol{u}^{(n+1)}\right)$ is defined pointwise almost everywhere at $\Gamma_{C}$.

The assertion of Lemma 2.1 follows directly from standart results on the local smoothness of the solution to mixed boundary value problems.

The stopping rule of Algorithm 1 is justified by the following considerations. If we assume that $A^{(n)}=A^{(n+1)}$, then from (2.7) we infer $\sigma_{33}\left(\boldsymbol{u}^{(n+1)}\right)<0$ at $A^{(n+1)}$, and $\llbracket u_{3}^{(n+1)} \rrbracket \geq 0$ at $I^{(n+1)}$. This, together with the conditions of (2.6) at $\Gamma_{C}$, proves that the iterates $\boldsymbol{u}^{(n+1)}$ and $A^{(n+1)}$ satisfy (2.5) if Algorithm 1 terminates in Step 3. In numerical experiments with properly chosen discretization, the algorithm typically terminates with $A^{(n)}=A^{(n+1)}$. If this was not the case, termination conditions based on residual must be used.

To investigate the behavior of Algorithm 1, first we introduce the concept of feasibility. We call the iterate $\boldsymbol{u}^{(n+1)}$ of (2.6) feasible if $\llbracket u_{3}^{(n+1)} \rrbracket \geq 0$ is satisfied at $\Gamma_{C}$. Second, we utilize a Papkovich-Neuber representation of the Lamé operator with the help of harmonic functions, which allow us to rely on a Hopf maximum principle. It enables 
us to state feasibility and a resulting monotonicity property of the iteration process.

Lemma 2.2. If an iterate $\boldsymbol{u}^{(n)}$ of (2.6) is feasible, then $\boldsymbol{u}^{(n+1)}$ is feasible, too, and

$$
\begin{gathered}
\llbracket u_{3}^{(n+1)} \rrbracket \geq \llbracket u_{3}^{(n)} \rrbracket \quad \text { on } \Gamma_{C}, \\
A^{(n+1)} \subseteq A^{(n)} .
\end{gathered}
$$

Proof. Let us denote by $\overline{\boldsymbol{u}}=\boldsymbol{u}^{(n+1)}-\boldsymbol{u}^{(n)}$. If $\llbracket u_{3}^{(n)} \rrbracket \geq 0$ at $\Gamma_{C}$, then the definition of $A^{(n)}$ according to (2.7) and the boundary conditions at $\Gamma_{C}$ according to (2.6) imply that $\llbracket \bar{u}_{3} \rrbracket=0$ at $A^{(n)}$ and

$$
p:=\sigma_{33}(\overline{\boldsymbol{u}})=-\sigma_{33}\left(\boldsymbol{u}^{(n)}\right) \leq 0 \quad \text { at } I^{(n)} .
$$

The difference of iterates (2.6) for $n+1$ and $n$ reads

$$
\begin{aligned}
& -\mu \Delta \overline{\boldsymbol{u}}-(\mu+\lambda) \nabla(\operatorname{div} \overline{\boldsymbol{u}})=\mathbf{0} \quad \text { in } \mathbb{R}^{3} \backslash \Gamma_{C}, \\
& \overline{\boldsymbol{u}}(\boldsymbol{x})=\boldsymbol{o}(1) \quad \text { as }|\boldsymbol{x}| \rightarrow \infty, \\
& \sigma_{13}(\overline{\boldsymbol{u}})=\sigma_{23}(\overline{\boldsymbol{u}})=0 \quad \text { on } \Gamma_{C}^{ \pm}, \\
& \llbracket \bar{u}_{3} \rrbracket(\overline{\boldsymbol{x}}, 0)=0 \quad \text { for } \overline{\boldsymbol{x}} \in A^{(n)}, \\
& \sigma_{33}(\overline{\boldsymbol{u}})(\overline{\boldsymbol{x}}, 0)=p(\overline{\boldsymbol{x}}) \quad \text { for } \overline{\boldsymbol{x}} \in I^{(n)} .
\end{aligned}
$$

For $\boldsymbol{x} \in \mathbb{R}_{+}^{3}:=\left\{\left(\overline{\boldsymbol{x}}, x_{3}\right) \in \mathbb{R}^{3}: x_{3}>0\right\}$ we determine the functions

$$
\begin{aligned}
& v_{\alpha}(\boldsymbol{x})=\bar{u}_{\alpha}\left(\overline{\boldsymbol{x}}, x_{3}\right)+\bar{u}_{\alpha}\left(\overline{\boldsymbol{x}},-x_{3}\right) \quad(\alpha=1,2), \\
& v_{3}(\boldsymbol{x})=\bar{u}_{3}\left(\overline{\boldsymbol{x}}, x_{3}\right)-\bar{u}_{3}\left(\overline{\boldsymbol{x}},-x_{3}\right),
\end{aligned}
$$

which satisfy the following relations at the boundary $\left\{x_{3}=0\right\}$ :

$$
v_{3}(\overline{\boldsymbol{x}}, 0)=\llbracket \bar{u}_{3} \rrbracket(\overline{\boldsymbol{x}}, 0), \sigma_{33}(\boldsymbol{v})(\overline{\boldsymbol{x}}, 0)=2 \sigma_{33}(\overline{\boldsymbol{u}})(\overline{\boldsymbol{x}}, 0) \text { for } \overline{\boldsymbol{x}} \in \mathbb{R}^{2} .
$$

With the help of (2.10) and (2.11) we can rewrite (2.9) for the function $\boldsymbol{v}=\left(v_{1}, v_{2}, v_{3}\right)^{\top}$ in $\mathbb{R}_{+}^{3}$ as

$$
\begin{aligned}
& -\mu \Delta \boldsymbol{v}-(\mu+\lambda) \nabla(\operatorname{div} \boldsymbol{v})=\mathbf{0} \quad \text { in } \mathbb{R}_{+}^{3}, \\
& \boldsymbol{v}(\boldsymbol{x})=\boldsymbol{o}(1) \quad \text { as }|\boldsymbol{x}| \rightarrow \infty, \\
& \sigma_{13}(\boldsymbol{v})=\sigma_{23}(\boldsymbol{v})=0 \quad \text { on }\left\{x_{3}=0\right\}, \\
& v_{3}(\overline{\boldsymbol{x}}, 0)=0 \quad \text { for } \overline{\boldsymbol{x}} \in \mathbb{R}^{2} \backslash I^{(n)}, \\
& \left(2 \mu v_{3,3}+\lambda \operatorname{div} \boldsymbol{v}\right)(\overline{\boldsymbol{x}}, 0)=2 p(\overline{\boldsymbol{x}}) \quad \text { for } \overline{\boldsymbol{x}} \in I^{(n)},
\end{aligned}
$$

where we used (2.1). The known Papkovich-Neuber representation of the mixed boundary-value problem (2.12) in a half-space expresses $\boldsymbol{v}$ 
in the form

$$
\begin{aligned}
& v_{\alpha}=(\kappa-1) U_{\alpha}-\kappa x_{3} U_{3, \alpha} \quad(\alpha=1,2), \\
& v_{3}=U_{3}-\kappa x_{3} U_{3,3} \quad\left(\kappa=\frac{\mu+\lambda}{2 \mu+\lambda}\right)
\end{aligned}
$$

by 3 harmonic potentials $\boldsymbol{U}=\left(U_{1}, U_{2}, U_{3}\right)^{\top}(\boldsymbol{x})$ such that $\Delta \boldsymbol{U}=\mathbf{0}$ and $\operatorname{div} \boldsymbol{U}=0$. As $x_{3}=0$ from (2.13) it follows that

$$
v_{3}(\overline{\boldsymbol{x}}, 0)=U_{3}(\overline{\boldsymbol{x}}, 0), \quad\left(2 \mu v_{3,3}+\lambda \operatorname{div} \boldsymbol{v}\right)(\overline{\boldsymbol{x}}, 0)=2 \mu \kappa U_{3,3}(\overline{\boldsymbol{x}}, 0)
$$

Thus the harmonic function $U_{3}$ satisfies the relations

$$
\begin{aligned}
& \Delta U_{3}=0 \quad \text { in } \mathbb{R}_{+}^{3} \\
& U_{3}(\boldsymbol{x})=o(1) \quad \text { as }|\boldsymbol{x}| \rightarrow \infty \\
& U_{3}(\overline{\boldsymbol{x}}, 0)=0 \quad \text { for } \overline{\boldsymbol{x}} \in \mathbb{R}^{2} \backslash I^{(n)} \\
& U_{3,3}(\overline{\boldsymbol{x}}, 0)=\frac{1}{\mu \kappa} p(\overline{\boldsymbol{x}}) \leq 0 \quad \text { for } \overline{\boldsymbol{x}} \in I^{(n)},
\end{aligned}
$$

hence $U_{3}(\overline{\boldsymbol{x}}, 0) \geq 0$ for $\overline{\boldsymbol{x}} \in \mathbb{R}^{2}$ due to the Hopf maximum principle. As a consequence of (2.11) and (2.14) we infer (2.8a). The feasibility of $\boldsymbol{u}^{(n+1)}$ and (2.7) leads to the assertion (2.8b). The proof is completed.

To attain the feasibility property, the initialization $A^{(-1)}=\emptyset$ in Step 0 of Algorithm 1 is suitable, as we shall see in the following lemma.

Lemma 2.3. For the iterate $\boldsymbol{u}^{(0)}$ satisfying

$$
\begin{array}{ll}
-\mu \Delta \boldsymbol{u}^{(0)}-(\mu+\lambda) \nabla\left(\operatorname{div} \boldsymbol{u}^{(0)}\right)=\boldsymbol{f} & \text { in } \mathbb{R}^{3} \backslash \Gamma_{C}, \\
\boldsymbol{u}^{(0)}(\boldsymbol{x})=\boldsymbol{o}(1) \quad \text { as }|\boldsymbol{x}| \rightarrow \infty, & \\
\sigma_{13}\left(\boldsymbol{u}^{(0)}\right)=\sigma_{23}\left(\boldsymbol{u}^{(0)}\right)=\sigma_{33}\left(\boldsymbol{u}^{(0)}\right)=0 & \text { on } \Gamma_{C},
\end{array}
$$

the next iterate $\boldsymbol{u}^{(1)}$ is feasible.

Proof. Denoting by $\overline{\boldsymbol{u}}=\boldsymbol{u}^{(1)}-\boldsymbol{u}^{(0)}$ with $\boldsymbol{u}^{(0)}$ from (2.16) we arrive at

$$
\begin{aligned}
A^{(0)} & =\left\{\overline{\boldsymbol{x}} \in \Gamma_{C}:\right. & & \left.\llbracket u_{3}^{(0)} \rrbracket(\overline{\boldsymbol{x}}, 0)<0\right\}, \\
I^{(0)} & =\left\{\overline{\boldsymbol{x}} \in \Gamma_{C}:\right. & & \left.\llbracket u_{3}^{(0)} \rrbracket(\overline{\boldsymbol{x}}, 0) \geq 0\right\} .
\end{aligned}
$$


Hence $\llbracket \bar{u}_{3} \rrbracket>0$ at $A^{(0)}$ and $p:=\sigma_{33}(\overline{\boldsymbol{u}})=0$ at $I^{(0)}$. Employing the representations (2.10) and (2.13) again, similarly to (2.15) we arrive at

$$
\begin{aligned}
& \Delta U_{3}=0 \quad \text { in } \mathbb{R}_{+}^{3}, \\
& U_{3}(\boldsymbol{x})=o(1) \text { as }|\boldsymbol{x}| \rightarrow \infty, \\
& U_{3}(\overline{\boldsymbol{x}}, 0)>0 \quad \text { for } \overline{\boldsymbol{x}} \in \mathbb{R}^{2} \backslash I^{(0)}, \\
& U_{3,3}(\overline{\boldsymbol{x}}, 0)=\frac{1}{\mu \kappa} p(\overline{\boldsymbol{x}})=0 \quad \text { for } \overline{\boldsymbol{x}} \in I^{(0)} .
\end{aligned}
$$

Utilizing the Hopf maximum principle, and assuming that there exists $\overline{\boldsymbol{x}}_{0} \in I^{(0)}$ such that $U_{3}\left(\overline{\boldsymbol{x}}_{0}, 0\right)<0$ attains its minimum, we obtain a contradiction to the condition $U_{3,3}\left(\overline{\boldsymbol{x}}_{0}, 0\right)=0$. Therefore, $U_{3}(\overline{\boldsymbol{x}}, 0) \geq 0$ at $I^{(0)}$. Thus, from $(2.11)$ and $(2.14)$, we derive $\llbracket u_{3}^{(1)} \rrbracket \geq \llbracket u_{3}^{(0)} \rrbracket \geq 0$ at $I^{(0)}$ and $\llbracket u_{3}^{(1)} \rrbracket=0$ at $A^{(0)}$.

Summarizing the results of Lemma 2.2 and Lemma 2.3, we conclude with the following proposition on global convergence.

Proposition 2.1. Starting with a feasible iterate $\boldsymbol{u}^{(n)}(n \geq 0)$, the iterates of Algorithm 1 are monotone, i.e.,

$$
\begin{gathered}
0 \leq \llbracket u_{3}^{(n)} \rrbracket \leq \llbracket u_{3}^{(n+1)} \rrbracket \leq \cdots \leq \llbracket u_{3}^{\star} \rrbracket \quad \text { on } \Gamma_{C}, \\
\Gamma_{C} \supseteq A^{(n)} \supseteq A^{(n+1)} \supseteq \cdots \supseteq A^{\star},
\end{gathered}
$$

for $\boldsymbol{u}^{\star}$ and $A^{\star}$ satisfying $(2.5)$

Proof. For the arbitrary, feasible iterate $\boldsymbol{u}^{(n)}$ with $\llbracket u_{3}^{(n)} \rrbracket \geq 0$ let us consider $\overline{\boldsymbol{u}}=\boldsymbol{u}^{\star}-\boldsymbol{u}^{(n)}$ with $\boldsymbol{u}^{\star}$ fulfilling (2.5). Using the relations (2.5) we derive $\llbracket \bar{u}_{3} \rrbracket(\overline{\boldsymbol{x}}, 0) \geq 0$ for $\overline{\boldsymbol{x}} \in A^{(n)}$, and $p(\overline{\boldsymbol{x}}):=\sigma_{33}(\overline{\boldsymbol{u}})(\overline{\boldsymbol{x}}, 0) \leq 0$ for $\overline{\boldsymbol{x}} \in I^{(n)}$. Therefore, the same arguments as used from (2.10) to (2.15) for $\overline{\boldsymbol{u}}$ imply $\llbracket \bar{u}_{3} \rrbracket \geq 0$ and, thus, (2.17a). Moreover,

$$
A^{\star} \cap I^{(n)} \subseteq\left\{\overline{\boldsymbol{x}} \in \mathbb{R}^{2}: \quad \llbracket u_{3}^{\star} \rrbracket(\overline{\boldsymbol{x}}, 0)=0, \quad \llbracket u_{3}^{(n)} \rrbracket(\overline{\boldsymbol{x}}, 0)>0\right\}=\emptyset,
$$

otherwise $\llbracket \bar{u}_{3} \rrbracket<0$ would yield a contradiction. The latter fact provides us with the assertion (2.17b).

\section{Discretized PRoblem AND NUMERICAL EXAMPleS}

\subsection{The primal-dual active set strategy after discretization.}

Here we return to the situation where $\Omega$ is a bounded domain and consider in the finite $N$-dimensional space the vector $\boldsymbol{X}=\left(X_{1}, \ldots, X_{N}\right)^{\top} \in$ $\mathbb{R}^{N}$ of unknowns assembling in an appropriate way components of the displacement vector $\left(u_{1}\left(\boldsymbol{x}^{m}\right), u_{2}\left(\boldsymbol{x}^{m}\right), u_{3}\left(\boldsymbol{x}^{m}\right)\right)^{\top}$ at grid points $\boldsymbol{x}^{m}$ with $m=(1, \ldots, N / 3)$ in the domain $\Omega_{C}$ with a crack. We assume that a 
jump $\llbracket u_{3} \rrbracket$ across the crack $\Gamma_{C}$ is described by differences $u_{3}\left(\boldsymbol{x}^{m}\right)-$ $u_{3}\left(\boldsymbol{x}^{k}\right)$ with $\boldsymbol{x}^{m} \in \Gamma_{C}^{+}$and $\boldsymbol{x}^{k} \in \Gamma_{C}^{-}$, thus forming a matrix $\boldsymbol{\Lambda} \in$ $\mathbb{R}^{|B| \times N}$ of a full column-rank $|B|<N$ for a set of indices $B$. We also suppose that the non-penetration condition reads $\boldsymbol{\Lambda} \boldsymbol{X} \geq \mathbf{0}$. Associated with a boundary traction $\sigma_{33}(\boldsymbol{u})$ at $\Gamma_{C}$, an unknown vector $\boldsymbol{Y}=\left(Y_{1}, \ldots, Y_{N}\right)^{\top} \in \mathbb{R}^{|B|}$ stands for dual variables (a Lagrange multiplier). For $\boldsymbol{F} \in \mathbb{R}^{N}$ given, after a suitable discretization of (2.2a)(2.2c), (2.3), and (2.4) we arrive at the linear complementarity problem:

$$
\begin{array}{r}
(\boldsymbol{\Lambda} \boldsymbol{X})_{m}=0 \quad \text { for all } m \in A=\left\{k \in B:(c \boldsymbol{Y}+\boldsymbol{\Lambda} \boldsymbol{X})_{k}<0\right\} \\
Y_{m}=0 \quad \text { for all } m \in I=\left\{k \in B:(c \boldsymbol{Y}+\boldsymbol{\Lambda} \boldsymbol{X})_{k} \geq 0\right\}
\end{array}
$$

with the stiffness matrix $\boldsymbol{L} \in \mathbb{R}^{N \times N}$ which is symmetric and positive definite.

Similar to Algorithm 1, a primal-dual active-set strategy applied to (3.1) implies the following iteration.

\section{Algorithm 2.}

(0) Choose $A^{(-1)} \subset B$; set $n=-1$.

(1) Solve for $\left(\boldsymbol{X}^{(n+1)}, \boldsymbol{Y}^{(n+1)}\right) \in \mathbb{R}^{N} \times \mathbb{R}^{|B|}$ :

$$
\begin{aligned}
& \boldsymbol{L} \boldsymbol{X}^{(n+1)}+\Lambda^{\top} \boldsymbol{Y}^{(n+1)}=\boldsymbol{F}, \\
& \left(\boldsymbol{\Lambda} \boldsymbol{X}^{(n+1)}\right)_{m}=0 \text { for all } m \in A^{(n)}, \\
& Y_{m}^{(n+1)}=0 \text { for all } m \in I^{(n)}:=B \backslash A^{(n)} .
\end{aligned}
$$

(2) Compute the active set:

$$
A^{(n+1)}=\left\{k \in B:\left(c \boldsymbol{Y}^{(n+1)}+\boldsymbol{\Lambda} \boldsymbol{X}^{(n+1)}\right)_{k}<0\right\} .
$$

(3) If $A^{(n+1)}=A^{(n)}$ then STOP; else set $n=n+1$ and go to Step 1.

In (Hintermüller et al. 2004), the well-posedness of the linear problem (3.2) with fixed $n$ was investigated in the general context of positivedefinite matrices. Further, the locally superlinear convergence and sufficient conditions for a global convergence to the solution of (3.1) as $n$ increases were given. Based on the unconditional results of Section 2, in the following we present in details computational features of Algorithm 2 applied to a homogeneous isotropic solid with a planar crack.

Notice that Algorithm 2 is not immediately a discrete version of Algorithm 1. This is due to the fact that system (3.2) represents a discrete version of the elasticity problem with certain settings on $A^{(n)}$ and $I^{(n)}$ on the bounded domain $\Omega_{C}$. However, if the far field influence 
is small, then the stiffness matrix $\boldsymbol{L}$ is close to the discrete operator of the problem on the unbounded domain.

3.2. Geometric and physical data of the problem. Let us treat Example 2 from Section 1 as the basic example for this and the next sections. We consider the unite cube $\Omega=\left\{0<x_{1}<1,0<x_{2}<\right.$ $\left.1,\left|x_{3}\right|<0.5\right\}$ containing inside the crack $\Gamma_{C}=\left\{0<x_{1}<0.75,0<\right.$ $\left.x_{2}<1, x_{3}=0\right\}$. We assume a volume load $\boldsymbol{f}=\mathbf{0}$, that the solid occupying the domain $\Omega_{C}=\Omega \backslash \Gamma_{C}$ is clamped at $\left\{x_{1}=1,0<x_{2}<\right.$ $\left.1,\left|x_{3}\right| \leq 0.5\right\}$, and that it is loaded by a traction force at $S=S^{+} \cup S^{-}$. The remain part of the boundary of $\Omega$ is assumed to be stress-free. The boundary loading is taken as

$$
\begin{array}{r}
-\sigma_{12}(\boldsymbol{u})=-g, \quad-\sigma_{22}(\boldsymbol{u})=0, \quad \sigma_{32}(\boldsymbol{u})=0 \\
\text { on } S^{ \pm}=\left\{0<x_{1}<0.9, x_{2}=0,0.1< \pm x_{3}<0.5\right\}
\end{array}
$$

with the constant $g=0.001 \mu$, Lamé parameters $\lambda=2 \nu \mu /(1-2 \nu)$ and $\mu=0.5 E /(1+\nu)$, where $\nu=0.34$ and $E=73000(\mathrm{mPa})$, thus $g \approx 27(\mathrm{mPa})$. Here $\mathrm{mPa}$ stands for mega Pascal. In Example 1 and Example 3 of Section 1, instead of (3.4) we had:

$$
\begin{aligned}
& -\sigma_{13}(\boldsymbol{u})=\mp g, \quad-\sigma_{11}(\boldsymbol{u})=-\sigma_{12}(\boldsymbol{u})=0 \\
\text { on } S^{ \pm}= & \left\{x_{1}=0,0<x_{2}<1,0.1< \pm x_{3}<0.5\right\},
\end{aligned}
$$

and

$$
\begin{array}{r}
-\sigma_{22}(\boldsymbol{u})= \pm g, \quad-\sigma_{12}(\boldsymbol{u})=-\sigma_{23}(\boldsymbol{u})=0 \\
\text { on } S^{ \pm}=\left\{0<x_{1}<0.9, x_{2}=0.5 \mp 0.5,0.1< \pm x_{3}<0.5\right\} .
\end{array}
$$

For the current feasibility study, we discretize the domain $\Omega_{C}$ with a crack by a uniform triangulation and apply standard linear finite elements. Accounting double points located symmetrically at the interface with crack $\left\{0<x_{1}<1,0<x_{2}<1, x_{3}=0\right\}$, the number $N$ of unknowns of $\boldsymbol{X}$ for the three-dimensional elasticity problem (3.1) is presented in Table 1 in dependence of the mesh-size $h$.

\begin{tabular}{|c|c|c|c|}
\hline mesh-size $h$ & 0.05 & 0.025 & $0.01(6)$ \\
\hline$\#$ unknowns $N$ & 29106 & 211806 & 692106 \\
\hline
\end{tabular}

TABLE 1. Number of unknowns $N$ for mesh-size $h$.

To solve the matrix equation (3.2), inner loop SSOR-iterations are terminated with tol $=10^{-10}$. For the determination of the active set in (3.3), a constant $c$ is taken in the range of $\left(10^{-3}, 10^{-2}\right)$. In the case 
when $c \boldsymbol{Y}^{(n+1)}+\boldsymbol{\Lambda} \boldsymbol{X}^{(n+1)}$ is close to zero, for computational reasons it can be helpful to use instead of (3.3):

$$
A^{(n+1)}=\left\{k \in B:\left(c \boldsymbol{Y}^{(n+1)}+\boldsymbol{\Lambda} \boldsymbol{X}^{(n+1)}\right)_{k}<-\delta\right\}
$$

with small $\delta>0$. In our examples we used $\delta=10^{-10}$.

3.3. Convergence of the numerical algorithm. Now we present the results of our numerical computations obtained by Algorithm 2 when solving problem (3.1) with the data from Section 3.2. In the following $h$ is fixed as $h=0.01(6)$.
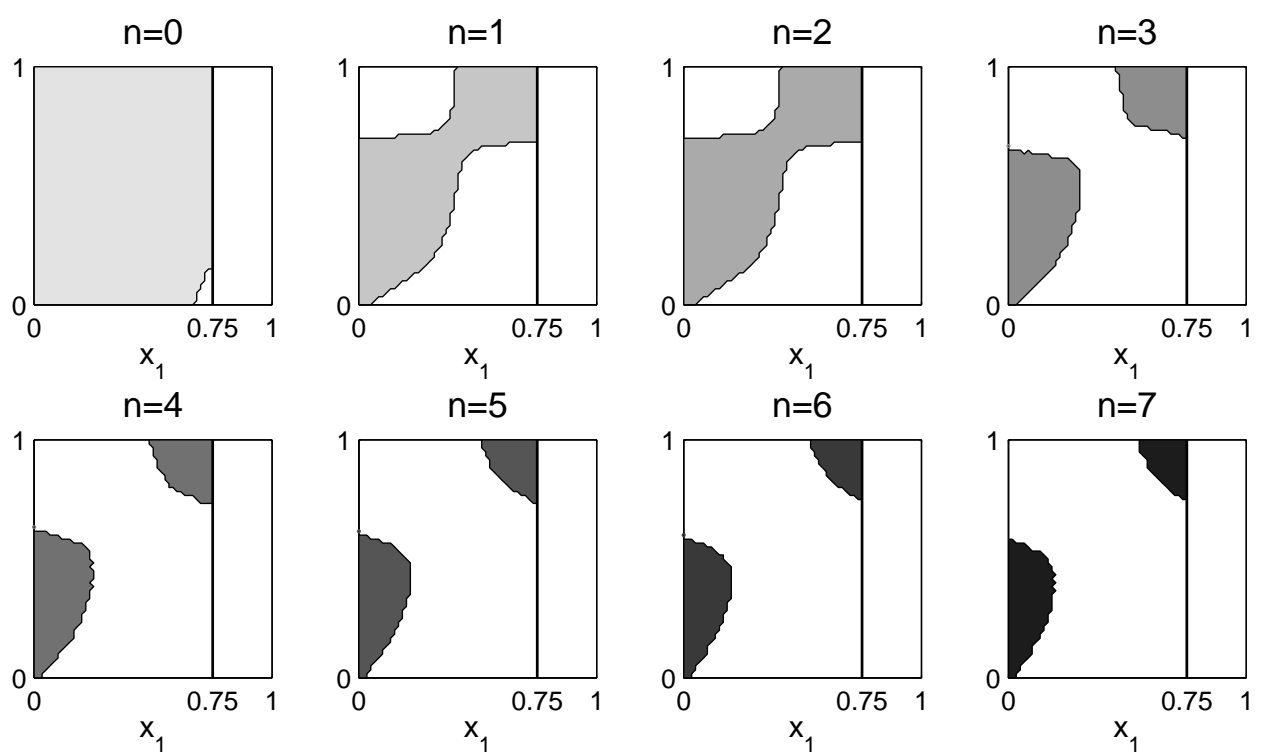

FiguRE 9. Iterations $A^{(n)}$ of the active set.

Starting with $A^{(-1)}=\emptyset$, the history of iterates $A^{(n)}$ of the active set as $n=0, \ldots, 7$ is illustrated in Figure 9. Algorithm 2 was terminated in Step 3 with the same active-set structure $A^{(n+1)}=A^{(n)}$ at iteration $n=$ 7 (i.e., after 9 iterates), thus reaching the exact solution $\left(\boldsymbol{X}^{\star}, \boldsymbol{Y}^{\star}, A^{\star}\right)=$ $\left(\boldsymbol{X}^{(n)}, \boldsymbol{Y}^{(n)}, A^{(n)}\right)$ to the discrete problem (3.1). During the iteration $A^{(0)}$ is split into two separate subsets. In Figure 9 we observe monotone convergence of active sets:

$$
\Gamma_{C} \supseteq A^{(1)} \supseteq \cdots \supseteq A^{(n)}=A^{(n+1)}:=A^{\star},
$$

which is in accordance with Proposition 2.1.

The history of the iterates $\llbracket u_{3}^{(n)} \rrbracket$ (respectively $\boldsymbol{\Lambda} \boldsymbol{X}^{(n)}$ ) of the jump of the displacements at the $\left\{x_{3}=0\right\}$-plane containing the crack $\Gamma_{C}$ is 

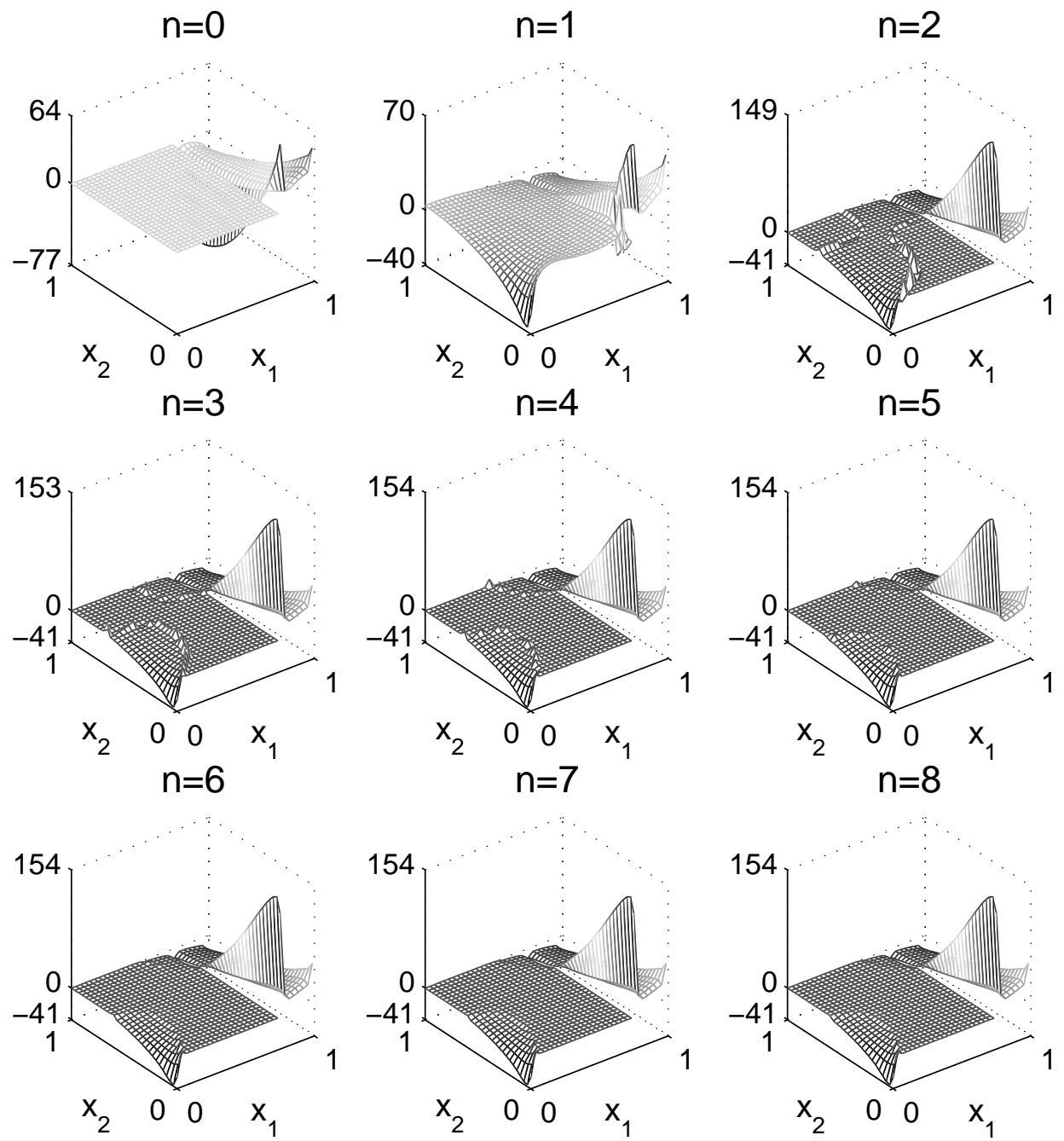

FiguRE 10. Iterates $\sigma_{33}\left(\boldsymbol{u}^{(n)}\right)$ of the stress at $\left\{x_{3}=0\right\}$.

depicted in Figure 11. The corresponding stress $\sigma_{33}\left(\boldsymbol{u}^{(n)}\right)$ reconstructed from the discrete Lagrange multiplicator $\boldsymbol{Y}^{(n)}$ by means of a piecewise linear approximation is presented in Figure 10.

Note that, for such initializations, problem (3.2) at $n=0$ represents an elasticity problem in the linear setting of stress-free crack surfaces without non-penetration conditions. Firstly, the corresponding active set $A^{(0)}$ implies mutual interpenetration between the crack surfaces, as it can be viewed in Figure 11, which is inconsistent physically. Secondly, 

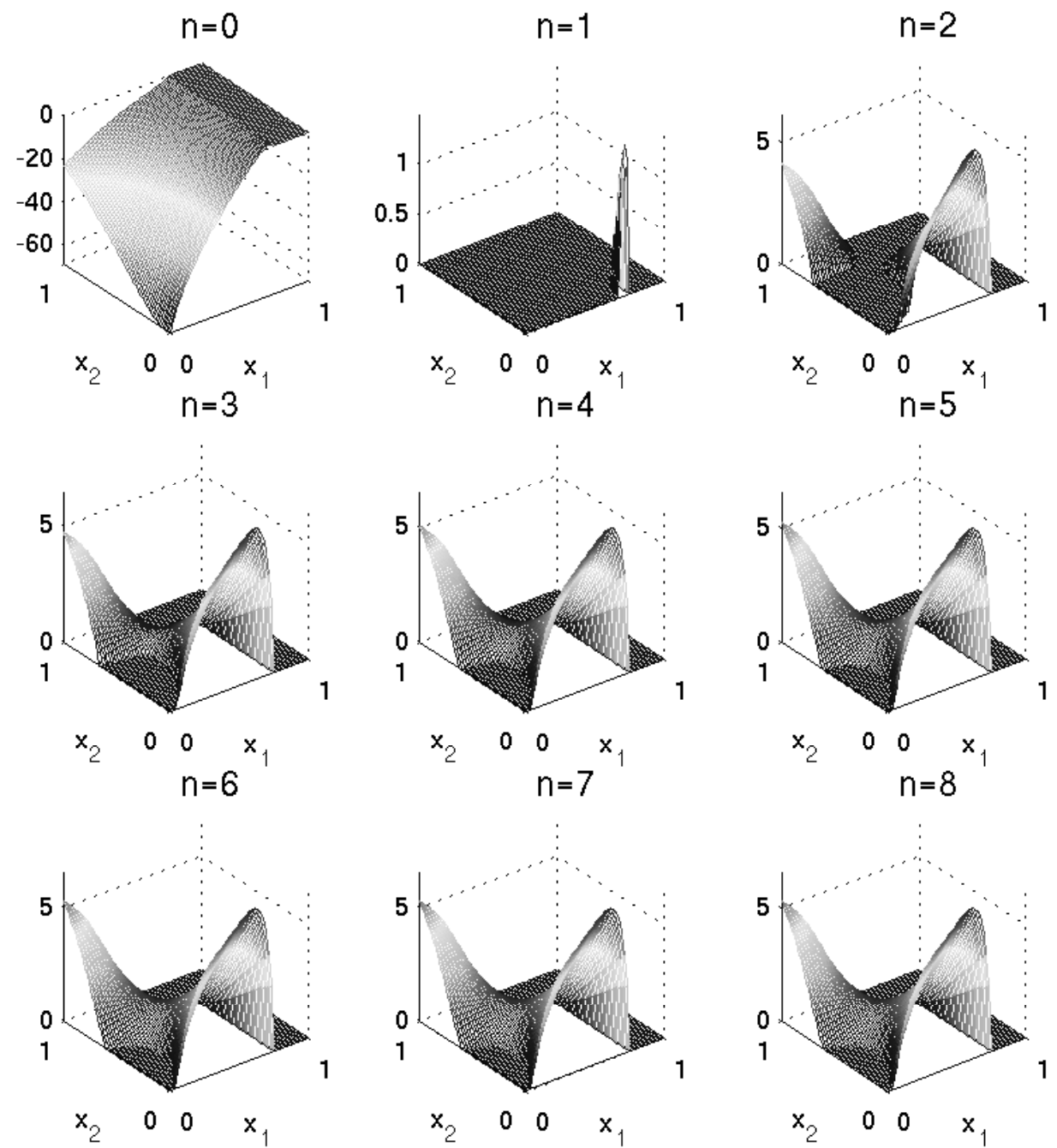

Figure 11. Iterates $\llbracket u_{3}^{(n)} \rrbracket \times 10^{5}$ of the jump at $\left\{x_{3}=0\right\}$.

$A^{(0)}$ differs significantly from the true active set $A^{\star}$ depicted in the last plot of Figure 9.

By refining the mesh, i.e. decreasing the mesh-size $h$, the numerical solutions to (3.1) computed by Algorithm 2 for various $h$ are compared with respect to the potential energy and the active set in Figure 12 (a) and (b), respectively. We observe linear convergence of the corresponding energies and that the actives sets are close to each other. 

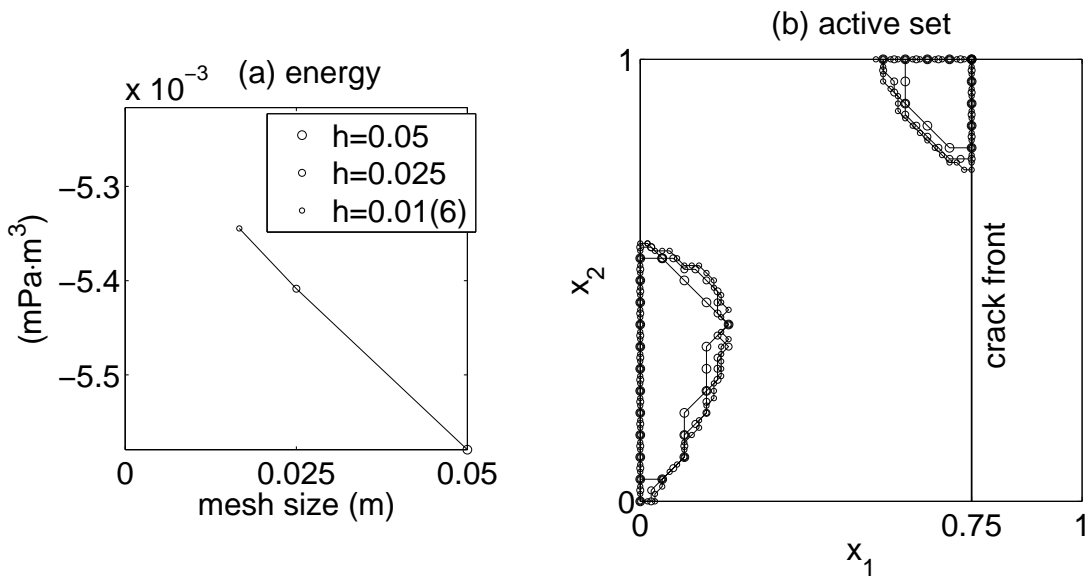

FIGURE 12. Solution characteristics by decreasing $h$.

The number of iterations required to successfully terminate Algorithm 2 is presented in Table 2 . Notice that it increases moderately with decreasing $h$.

\begin{tabular}{|l|c|c|c|}
\hline mesh-size $h$ & 0.05 & 0.025 & $0.01(6)$ \\
\hline$\#$ iterations & 7 & 8 & 9 \\
\hline
\end{tabular}

TABle 2. Number of iterations for mesh-size $h$.

The last two facts illustrate a stable behavior of the primal-dual active-set algorithm also with respect to the mesh refinement.

\section{Discussion}

In this paper a primal-dual active set strategy is proposed to numerically solve crack problems with non-penetration conditions. We focus on three-dimensional aspects. On the basis of this approach we present some of our findings in numerical experiments.

To interpret the physical implications we observe the following. The stress intensity factors $K_{2}$ and $K_{3}$ are not influenced significantly when frictionless contact occurs at the crack. On the contrary, $K_{1}$ can take a negative value due to the contact implying self-penetration of crack faces. It happens often in the engineering practice that a small $K_{1}<0$ can be ignored without essential loss of accuracy. However, we find that the error in $K_{1}$ can be rather large in the following cases: First, it is evident that the pure compression loading gives rise to contact between the opposite crack faces. Secondly, for arbitrary loading applied at the 
boundary of a finite body the compression traction at a crack cannot be neglected in three spatial dimensions. In our examples we demonstrate that boundary effects have a significant influence on the stress intensity factors. Thirdly, applying the commonly adopted simplification due to plane deformations disregards the three-dimensional nature of contact at a crack.

Acknowledgment. The research results were obtained with the support of the Austrian Science Fund (FWF) in the framework of the START-program Y305 "Interfaces and Free Boundaries", the research project P18267-N12, the SFB F32 "Mathematical Optimization and Applications in Biomedical Sciences", and the Russian Foundation for Basic Research (project 06-01-00209). We thank I.I. Argatov from St.Petersburg for useful discussions on the mechanics.

\section{REFERENCES}

Aliabadi, M.H., Rooke, D.P., 1992. Numerical Fracture Mechanics. Kluwer, Southampton.

Cherepanov, G.P., 1979. Mechanics of Brittle Fracture. McGraw-Hill, New York.

Cisilino, A.P., Aliabadi, M.H., 1999. Three-dimensional boundary element analysis of fatigue crack growth in linear and non-linear fracture problems. Engng. Fract. Mech. 63, 713-733.

Geubelle, P.H., Rice, J.R., 1995. A spectral method for three-dimensional elastodynamic fracture problems. J. Mech. Phys. Solids 43, 1791-1824.

Goldstein, R.V., Entov, V.M., 1994. Qualitative Methods in Continuum Mechanics. Longman, Harlow; Wiley, New York.

Gosz, M., Dolbow, J., Moran, B., 1998. Domain integral formulation for stress intensity factor computation along curved three-dimensional interface cracks. Int. J. Solids Structures 35, 1763-1783.

Hintermüller, M., Ito, K., Kunisch, K., 2003. The primal-dual active set strategy as a semismooth Newton method. SIAM J. Optim. 13, 865-888.

Hintermüller, M., Kovtunenko, V.A., Kunisch, K., 2004. The primal-dual active set method for a crack problem with non-penetration. IMA J. Appl. Math. 69, $1-26$.

Hu, G.-D., Panagiotopoulos, P.D., Panagouli, O.K., Scherf, O., Wriggers, P., 2000. Adaptive finite element analysis of fractal interfaces in contact problems. Comput. Meth. Appl. Mech. Engng. 182, 17-37.

Ito, K., Kunisch, K., 2003. Semi-smooth Newton methods for the variational inequalities of the first kind. ESAIM Math. Modelling Numer. Anal. 37, 41-62.

Kachanov, M., Karapetian, E., 1997. Three-dimensional interaction of a half-plane crack with point forces, dipoles and moments. Int. J. Solids Structures 34, 41014125 .

Khludnev, A.M., Kovtunenko, V.A., 2000. Analysis of Cracks in Solids. WIT-Press, Southampton, Boston. 
Khludnev, A.M., Sokolowski, J., 1999. The Griffith formula and the CherepanovRice integral for crack problems with unilateral conditions in nonsmooth domains. Euro. J. Appl. Math. 10, 379-394.

Kovtunenko, V.A., 2006a. Primal-dual sensitivity analysis of active sets for mixed boundary-value contact problems. J. Engineering Math. 55, 151-166.

Kovtunenko, V.A., 2006b. Interface cracks in composite orthotropic materials and their delamination via global shape optimization. Optimiz. Engng. 7, 173-199.

Morozov, N.F., Petrov, Yu.V., 2000. Dynamics of Fracture. Springer, Berlin.

Nikishkov, G.P., Atluri, S.N., 1987. Calculation of fracture mechanics parameters for an arbitrary three-dimensional crack, by the 'equivalent domain integral' method. Int. J. Numer. Meth. Engng. 24, 1801-1821.

Stavroulakis, G.E., 2001. Inverse and Crack Identification Problems in Engineering Mechanics. Kluwer, Dordrecht.

Sukumar, N., Moës, N., Moran, B., Belytschko, T., 2000. Extended finite element method for three-dimensional crack modelling. Int. J. Numer. Math. Engng. 48, 1549-1570.

Zozulya, V.V., Menshykov, O.V., 2003. Use of the constrained optimization algorithms in some problems of fracture mechanics. Optimiz. Engng. 4, 365-384. 